\title{
Validity of county-level estimates of climate change beliefs
}

To the Editor - In their recent Letter in Nature Climate Change, Howe et al ${ }^{1}$. draw attention to the scientific and policy importance of local-scale data on public perceptions about climate change. Climate perceptions exhibit place-to-place variation ${ }^{2}$, but local-scale social data often do not exist. To better resolve local variations, Howe and co-authors present results from downscaling methods applied to national surveys to characterize perceptions in US states, 435 congressional districts and 3,143 counties. Their validation exercise compares downscaled results with direct estimates from surveys asking a related climate change question.

Here we undertake a broader validation exercise using independent survey data from 30 US counties. Howe et al. caution that their method understates place-to-place variation, and our results reinforce this, but we also find evidence that their estimates are unbiased and moderately correlated with direct measurements even at smaller scales.

A general question about climate change beliefs (Supplementary Information) was asked as part of surveys by the Community and Environment in Rural America (CERA) and Communities and Forests in Oregon (CAFOR) projects that targeted selected and often non-metropolitan counties ${ }^{3-5}$. Figure 1 illustrates belief in anthropogenic climate change as estimated by Howe et al. against direct measures from 30 counties where CERA/CAFOR completed at least 100 interviews. The +0.46 correlation $(p<0.05)$ seems encouraging given the expected imprecision of the Howe et al. method for small geographies, compounded by sampling variation in CERA/CAFOR. For 17 of these counties the estimates fall within CERA/CAFOR confidence intervals.

Across the 30 counties we analysed, percentages from CERA/CAFOR surveys and Howe et al. have similar unweighted means -47 and 46 respectively. This is in accordance with nationwide comparisons (Supplementary Information) and supports the view that the county-level estimates of Howe et al. are unbiased. But Fig. 1 highlights a systematic difference. CERA/ CAFOR percentages exhibit greater range (30 versus 17 ) and much greater variance

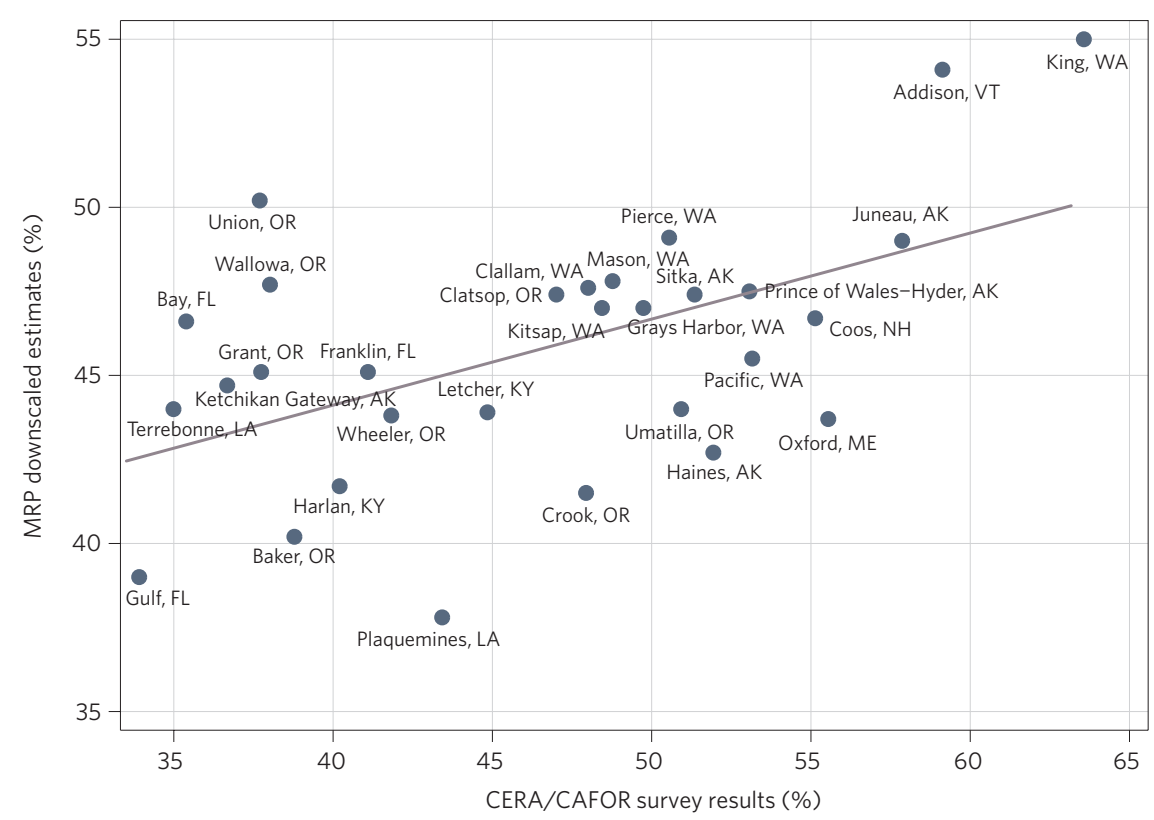

Figure 1 | Public acceptance of anthropogenic climate change in 30 US counties. Multilevel regression with poststratification (MRP) estimates are shown versus CERA/CAFOR survey results. Question wording and national comparisons are given in the Supplementary Information.

(63 versus 12). A Monte Carlo experiment suggests that sampling error plausibly makes the observed variance of CERA/CAFOR values about $14 \%$ higher compared with the (unknown) variance of population belief in anthropogenic climate change in these counties. This implies that Howe et al. estimates exhibit about 78\% less variance than the true population belief.

As Howe et al. note, substantial reductions from the true variance are expected from regression-prediction methods, particularly in small samples. Moreover, because the variance found by Howe et al. depends partly on prediction by location-level demographics or geographical effects, correlations between estimated climate perceptions and other demography/geography-related place characteristics could be artificially inflated.

Keeping these caveats in mind, although variance attenuation and regression-based estimation complicate modelling with finescale data, the apparent lack of bias and moderate correlation with directly observed percentages encourages further analysis.
References

1. Howe, P. D., Mildenberger, M., Marlon, J. R. \& Leiserowitz, A Nature Clim. Change 5, 596-603 (2015).

2. Hamilton, L. C. \& Keim, B. D. Int. J. Climatol. 29, 2348-2352 (2009).

3. Hamilton, L. C., J. Hartter, J., Safford, T. G. \& Stevens, F. R. Rural Sociol. 79, 257-281 (2013).

4. Hamilton, L. C. \& Safford, T. G. Soc. Natur. Resour. 28, 57-74 (2015).

5. Boag, A. et al. Forest Views: Shifting Attitudes Toward the Environment in Northeast Oregon (Carsey School of Public Policy, Univ. New Hampshire, 2015).

Additional information

Supplementary information is available in the online version of the paper.

Lawrence C. Hamilton', Joel Hartter ${ }^{2}$ and Thomas G. Safford ${ }^{1}$

${ }^{1}$ Sociology Department, University of New Hampshire, Durham, New Hampshire 03824, USA. ${ }^{2}$ Environmental Studies Program, University of Colorado, Boulder, Colorado 80309, USA.

e-mail: Lawrence.Hamilton@unh.edu 


\section{Correction}

In the version of the Correspondence 'Validity of county-level estimates of climate change beliefs' originally published (Nature Clim. Change 5, 704; 2015), the correlation coefficient should have read +0.46 $(p<0.05)$, not $+0.53(p<0.01)$. Further, the number of counties where estimates fall within CERA/CAFOR confidence intervals is 17, not 18 . This changes the variance figures to 63 vs. 12 (not 15), which suggests that the estimates by Howe etal. exhibit about 78\% (not 74\%) less variance than the true population belief. These calculation errors were made as a consequence of a coding error. These errors have been corrected and new Supplementary Information files uploaded online 13 October 2015. 